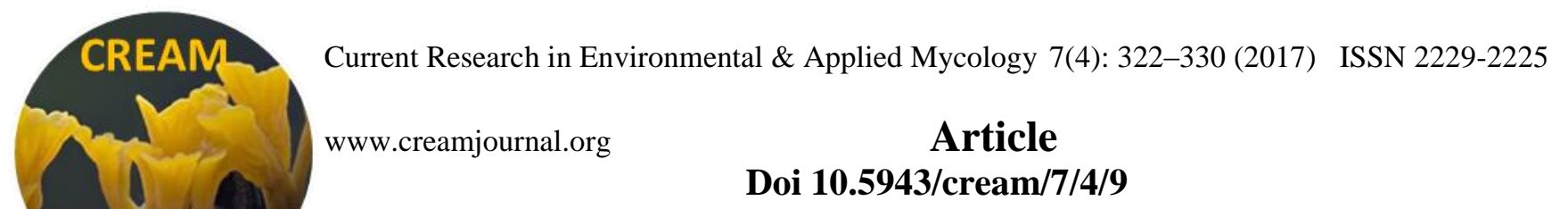

Copyright $(\odot)$ Beijing Academy of Agriculture and Forestry Sciences

\title{
Mycetozoan incidence in soils and their potential for ecosystem quality assessment
}

\author{
Guyer HE ${ }^{1}$, Rojas PA², Rollins AW', Rojas $\mathbf{C}^{2,4}$
}

${ }^{1}$ Department of Agricultural and Biosystems Engineering, Iowa State University, Ames, Iowa 50011, USA

${ }^{2}$ Engineering Research Institute, University of Costa Rica, San Pedro de Montes de Oca, 11501-Costa Rica

${ }^{3}$ Department of Biology and Cumberland Mountain Research Center, Lincoln Memorial University, Harrogate TN 37752, USA

${ }^{4}$ Department of Biosystems Engineering, University of Costa Rica, San Pedro de Montes de Oca, 11501-Costa Rica

Guyer HE, Rojas PA, Rollins AW, Rojas C 2017 - Mycetozoan incidence in soils and their potential for ecosystem quality assessment. Current Research in Environmental \& Applied Mycology 7(4), 322-330, Doi 10.5943/cream/7/4/9

\begin{abstract}
Two experiments employing a modified version of the standard "Cavender Method" were used to evaluate the incidence patterns of myxomycete and dictyostelids associated with different soils collected across north and central America. The soils were subjected to variable culturing conditions and parameters including plant material quality, agar type and bacterial food source. Ecological variables such as geographic location and land use quality were also evaluated to determine potential differences affecting the soils. The study also aimed to document the potential for mycetozoans to serve as indicators of ecosystem quality. The results indicated that plant materials with middle hardness and moderate cellulose to lignin ratio, in conjunction with an intermediate rich culturing media favoured the growth of mycetozoans. Also, Bacillus subtilis represented a suitable alternative to Escherichia coli. Dictyostelids were more commonly recovered from tropical soils than temperate soils, while the opposite pattern was observed for myxomycetes. No differences in mycetozoan incidence were detected when landscape-scale and soil quality parameters were examined. Overall, data related to the utility of using mycetozoans as bioindicators are still limited, but the results of this study suggest that more targeted, scaledependent studies are warranted. The modified protocol used herein appears to represent a reliable method to generate consistent data for ecological studies of mycetozoans, particularly when tropical soils are used.
\end{abstract}

Key words - applied microbiology - biosystems - ecology - dictyostelids - myxomycetes

\section{Introduction}

In recent decades, there have been an increasing number of studies evaluating the potential uses of mycetozoans across different fields of human knowledge (e.g. Tran et al. 2008, Herrera et al. 2011). Even though in the field of applied environmental microbiology, there are previous studies (e.g. Clissmann et al. 2015), the number of investigations carried out under this scope in the Neotropics is limited. This pattern partially corresponds to the fact that mycetozoan researchers across this geographic area have focused on the development of distributional patterns of species (see Lado et al. 2017), but also relates to their limited involvement with applied subdisciplines. 
Parallel to the latter, most modern mycetozoan researchers simply utilize existing isolation methods without exercising their own protocol development approach. As a consequence, there have been few contributions to the development of new isolation protocols during the last decades (one exception being Douglas et al. 2013). This is particularly true for myxomycetes. Ironically, a large number of ecological studies carried out in the last decades have used the microcosm approach to obtain data. Given the fact that microcosms, as an experimental strategy, are by design very sensitive to the control on variables (Daelher \& Strong 1996), it is important and necessary to revisit common procedures to study mycetozoan isolation in laboratory conditions.

The present study has been designed with the main objective of evaluating different laboratory isolation variables on the generation of data on mycetozoans and the potential use of such information to understand the capacity of two groups of organisms to indicate ecosystem quality. In this manner, the present investigation represents an opportunity for future research on mycetozoans in the fields of biodiversity and ecosystem monitoring.

The integration of basic information on mycetozoans with particular applications, such as the determination of ecosystem quality through microbial ecology, is relevant in the Neotropics. As mentioned, mycetozoans in this geographic area have been studied over the past decades, but the use of this information for environmental applications has been almost non-existent. The present study aimed to increase the body of information available in this context.

\section{Materials \& Methods}

This study was conducted between 2015 and 2016 in the Engineering Research Institute at the University of Costa Rica and the Cumberland Mountain Research Center at Lincoln Memorial University and consisted of two separate experiments. Each experiment addressed a unique research question, but they both utilized the same basic protocol applied to environmental soil samples. Only incidence data, quantified by myxomycete and dictyostelid life stages appearing in soil cultures, was recorded. Records were not identified to species and secondary isolations were not performed.

A single bulk soil sample (approximately 250 grams) was obtained from a randomly selected point within each of the 22 study sites. Eleven tropical sites 1) Miramar (MCR), 2) Bagaces (BCR), 3) Corredores (COCR), 4) Tierra Blanca (TBCR), 5) Coto Brus (CBCR), 6) Turrialba (TCR), 7) Cahuita (CACR), 8) San Carlos (SCCR), 9) Acosta (ACR), 10) Sarapiquí (SCR) and 11) San Pedro de Montes de Oca (SPCR) were sampled across Costa Rica. Likewise, 11 temperate sites 12) Big Stone Gap (BUS), VA, 13) Jenkins (JUS), KY, 14) Eolia (EUS), KY, 15) Kingdom Come (KCUS), KY, 16) Harlan (HUS), KY, 17) Pine Mountain (PMUS), KY, 18) Highland Park (HPUS) at Baton Rouge, LA, 19) Geranium St (GUS) at Baton Rouge, LA, 20) Indian Hill Plaza (IHUS) in Mount Pleasant, MI, 21) Isabella Rd (IRUS) in Mount Pleasant, MI and 22) Pickard Road (PRUS) in Mount Pleasant, MI were sampled in the United States of America.

Each soil sample was collected into a paper bag after removing the accumulated detritus and the first two centimeters of topsoil. The samples were air dried, homogenized by passing through a \#10 (2 mm) mesh screen soil sieve, and transported to the laboratory via airmail from the United States or by car in Costa Rica. In the lab a $4.5 \mathrm{~g}$ subsample of soil was extracted from each sample and mixed with $45 \mathrm{ml}$ of distilled water in a sterile container producing a 1:10 dilution. The slurry was mixed by hand for 15 minutes and then a $0.15 \mathrm{ml}$ aliquot was transferred to a $100 \times 15 \mathrm{~mm}$ Petri dish containing $1.5 \%$ hay infusion agar (Atlas 2004). Then a $0.15 \mathrm{ml}$ aliquot of a bacterial suspension (see below) grown in nutrient broth was then added to the dish. Finally, a bait (an autoclaved substrate with a mass of approximately $0.10 \mathrm{~g}$, see below) was placed on the surface of the agar. The bait was added in an attempt to promote myxomycete activity and represented a modification of "Cavender Method" (see Spiegel et al. 2004). All cultures were maintained for two weeks in a ventilated semi-sealed chamber in a climate-controlled room. Mycetozoan incidence was quantified on days seven and 15. Myxomycete incidence was recorded as presence of plasmodia or fruiting bodies in a culture plate, while dictyostelid incidence was recorded by the presence of slugs, or clones in a culture plate. 
Eight baits were selected to encompass a range of different structural and chemical characteristics. In order of structural integrity from hardest to softest, the baits were 1) five $\mathrm{mm}$ teak wood cubes, 2) coconut husks, 3) coconut bracts, 4) straw, 5) forest leaves, 6) coffee pulp, 7) Gmelina sp. sawdust, and 8) disaggregated cellulose. Two different types of bacteria Escherichia coli (a rod-shaped, gram-negative, facultative anaerobe) and Bacillus subtilis (a rod-shaped, grampositive, facultative anaerobe) were selected as food sources. While both species are commonly associated with the intestinal microflora of endotherms, they are frequently encountered in soils where they are introduced through fecal deposition.

Experiment one was designed 1) to test the effect of different bacteria and bait combinations on the generation of mycetozoan incidence data from soil cultures and 2) to subsequently examine potential differences in mycetozoan incidence between soils obtained from tropical and temperate regions. To do this, two full runs of duplicate sample isolates (prepared as described above) were generated using all combinations from the matrix formed by the 22 soil samples and eight baits. The complete set of two runs was repeated twice by using the two bacteria tested, Escherichia coli and Bacillus subtilis. In order to decrease potential biases due to temporal effects, the complete experiment was executed in batches representing the different experimental units, which were spatially and temporally arranged in a random fashion.

Experiment one also utilized both ten replicates of a positive (a 1:10 soil dilution plated on hay-infusion agar, with a bacterial suspension within the standard of the Cavender method, but no bait) and 10 replicates of a negative control (a bacterial suspension plated on hay-infusion agar and a bait, but no 1:10 soil dilution). The positive control was used to validate that the system was functional (i.e., mycetozoans could be cultured) while the negative control was used to validate that mycetozoans were not derived from the agar, bacterial suspension, or the bait. Collectively, the complete experimental model consisted of 704 individual experimental units, excluding controls and tested all combinations of soil, substrate, and bacterium types.

The second experiment was designed to 1) test differences in mycetozoan incidence in soils among disturbance types and geographical locations along the gradient from Michigan, USA to Costa Rica and 2) test the effect of different enrichment treatments for the generation of mycetozoan incidence data from a standardized soil isolation protocol in the laboratory. In a similar manner to experiment one, a complete model composed of a total of 336 individual experimental units, excluding controls, was generated by using 14 soil samples, two baits, two bacteria types and three types of media with different enrichment levels. The latter corresponded to $1.5 \%$ water agar (Riedelde Haën), cornmeal agar (Carolina Biological Supply) and potato-dextrose agar (Riedel-de Haën). For this experiment, only one complete run was conducted.

This experiment utilized soil samples from three of the locations in the previous experiment but used samples from additional locations in Mexico and Costa Rica. The previously utilized soil samples corresponded with IHUS, IRUS, and PRUS in Michigan, BUS, EUS, and PMUS in Kentucky, and HPUS and GUS in Louisiana. Additional soil samples were obtained from three locations in the Yucatan Peninsula in Mexico and corresponded with the Merida Town Central Park (MMX), the coastal town of Progresso (PMX), and the town of Dzibilchaltun (DMX). Similarly, additional soil samples were collected in Costa Rica and corresponded with the buffer area of the Tapanti National Park (TACR), the town of Orosi (OCR), and the Technological Institute of Costa Rica (TECR, see Fig. 1).

In order to evaluate relationships between incidental data and micro and macroenvironmental characteristics associated with each location, a series of extra parameters were measured or calculated in the second experiment. A GIS-based categorization of land use was developed using Landsat 8 images for a 1-mile section surrounding all 14 sampled locations. Two land use categories, forest cover and houses, were used to classify differences in the level of ecosystem disturbance. As such, three distinct categories were created. Low disturbance was characterized by the highest percentage of forest cover $(>50 \%)$ and lowest of houses $(<1 \%)$. Intermediate disturbance had low forest cover (average of 12.4\%) and intermediate house cover (between 5\% and 15\%). High disturbance had low forest cover $(<13 \%)$ and highest house cover $(>15 \%)$. The average Normalized 
Difference Vegetation Index (NDVI) was calculated for each location as well (Gates 1980). Soil texture was quantified (via triplicate samples) based on the percentage composition of sand, silt, and clay. Additionally, water retention capacity calculated (via triplicate samples) as mililiters of water retained per gram of soil after soil samples were dried at $60^{\circ} \mathrm{C}$ for 48 hours.

All statistical analyses were carried out using the programs JMP v10.0 or PAST v3.12. First, normality was checked (using the Shapiro-Wilk test). Parametric estimators were used when the assumption of normality was not violated and non-parametric estimators when the data failed to reasonably approximate a normal distribution. In all cases, single relationships between variables under study and total number of records from the presence/absence recording were tested using the contingency table approach or analysis of variance when appropriate. In all statistical analyses, outliers (defined herein as values beyond the 25-75 interquartile range) were excluded and an alpha of 0.05 was used as a cut-off value for the rejection of the null hypothesis. The intuitive (1-D) Simpson Diversity Index was calculated and followed up by a t-test in selected comparisons in order to evaluate potential differences across levels of analyses.

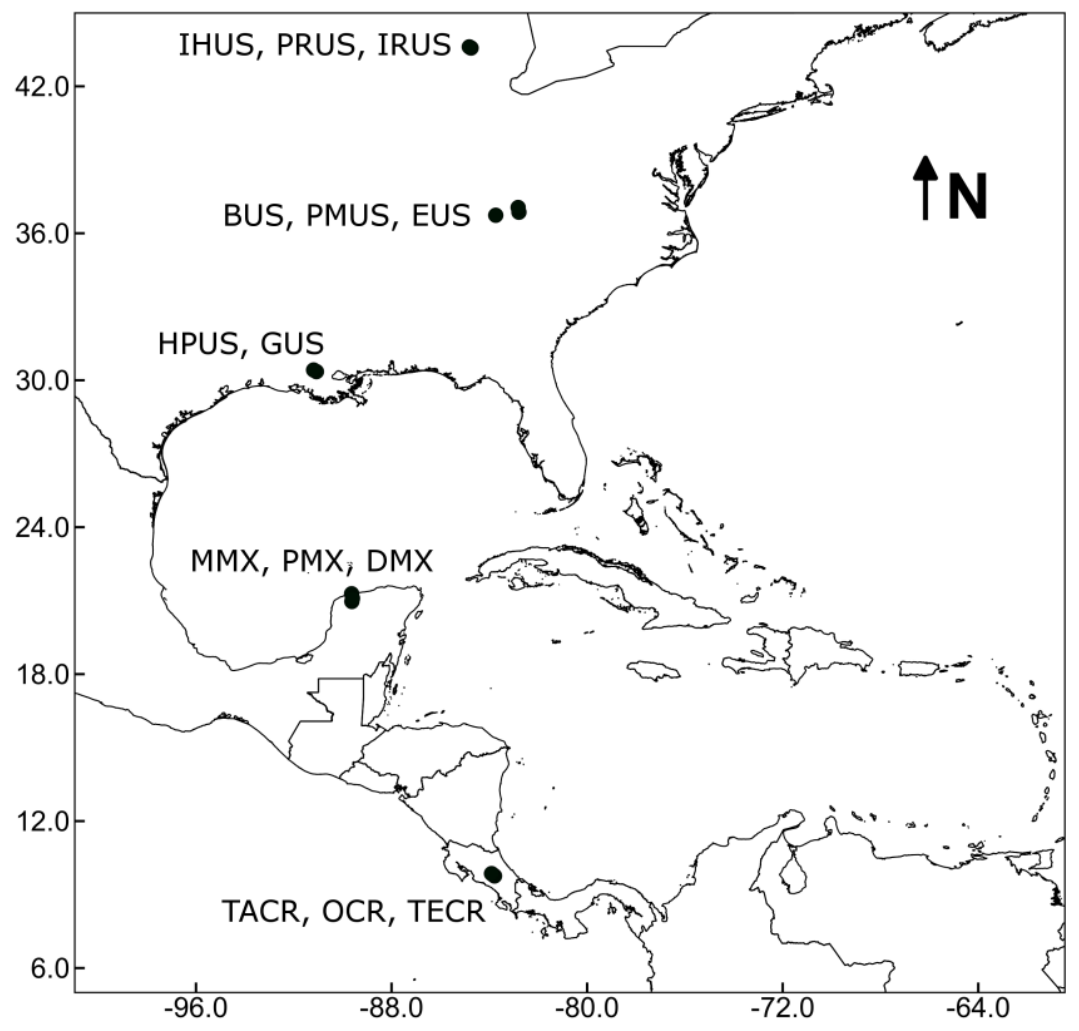

Fig. 1 - Section of the Americas showing the locations from where soil samples used in experiment two were collected.

\section{Results}

The first experiment determined that soil cultures using $B$. subtilis as a bacterial food source resulted in a higher incidence of myxomycetes relative to $E$. coli than expected by chance alone $\left(\chi^{2}=4.27\right.$, d.f. $\left.=1, P=0.04\right)$ and results from the logistic model indicated that $B$. subtilis was not favorable for dictyostelids $\left(\chi^{2}=62.39, d . f .=1, P=0.0001\right)$. The incidence of dictyostelids and myxomycetes varied according to the type of bacterium used in the isolation protocol and the origin of the soil (Fig. 2). The highest incidence values were always observed in tropical soils, with larger differences recorded for dictyostelids than for myxomycetes. However, myxomycete incidence from temperate soils was moderate to high (see Fig. 2, higher than 30\%) in this experiment.

No statistical difference was found in the incidence according to bait type when evaluated either by totals (bacterial types pooled) or by total values per bacterium (see Fig. 3, for bacterial 
types pooled). This lack of differences was observed for both the temperate and the tropical datasets individually as well. However, the highest values of myxomycete incidence were recorded from tropical soil samples using straw or forest leaves as baits; whereas for dictyostelids, the most productive baits were coconut bracts and straw and the highest incidence was associated with tropical soils. Also, no statistically significant difference was found for the incidence of myxomycetes between temperate and tropical areas $(t=-1.90, d . f .=175, P=0.06)$. However, there was a difference in the incidence values of dictyostelids between temperate and tropical areas ( $t=-$ 8.21, d.f. $=175, P=0.0001)$, with Costa Rica showing the highest values. This difference was observed both when $E$. coli or $B$. subtilis were used $(F(1,21)=9.18, P=0.0066$ and $F(1,21)=12.94$, $P=0.0018$, respectively) and was also recorded by means of the Simpson Diversity Index $(t=2.81$, $d . f .=52, P=0.007)$.

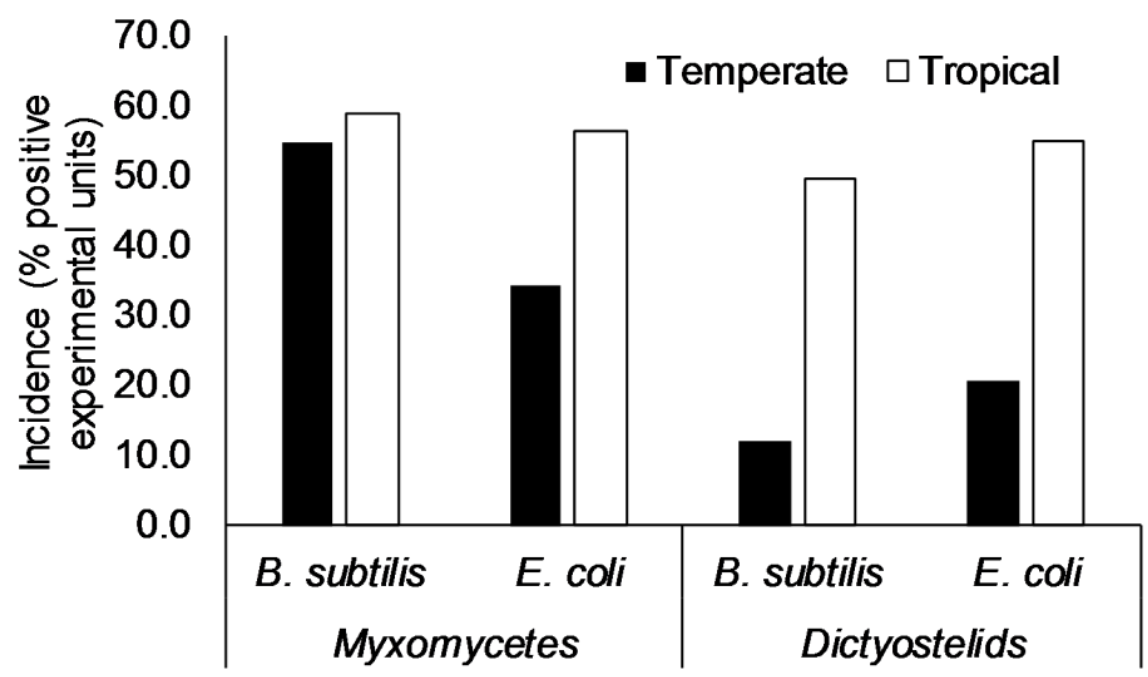

Fig. 2 - Distribution of the number of positive experimental units (incidence) recorded in experiment one according to bacterium and mycetozoan type. $\mathrm{N}=704$.

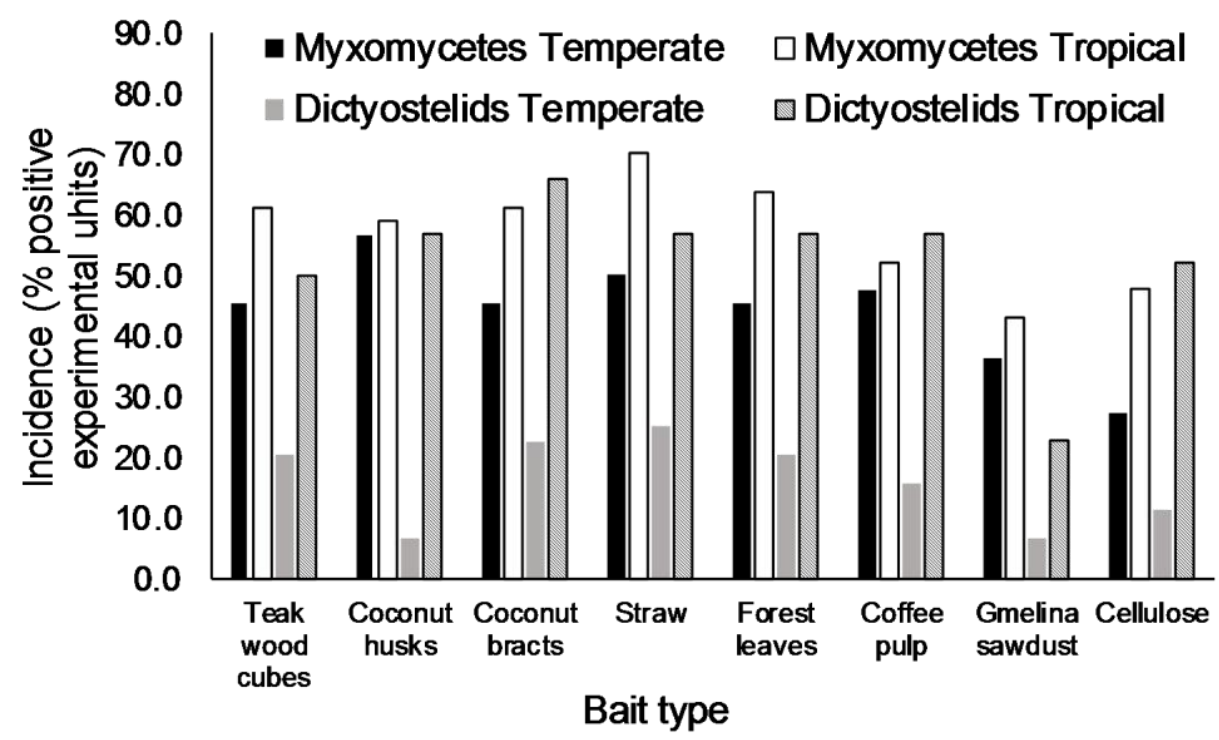

Fig. 3 - Distribution of the number of positive experimental units (incidence) recorded in the present study according to bait types, mycetozoan group and geographical location. $\mathrm{N}=704$. 
Differences in the incidence of myxomycetes $\left(\chi^{2}=40.98, d . f .=21, P=0.005\right)$ and dictyostelids $\left(\chi^{2}=58.96\right.$, d.f. $\left.=18, P=0.0001\right)$ by soil sample, were recorded. Both EUS and ACR showed the lowest myxomycete incidence values, whereas two of the temperate locations (PRUS and IRUS) were among the sites with the highest myxomycete incidence values. For dictyostelids, three locations in the tropical setting showed the highest values (SCR, SCCR, and SPCR) and two locations in the temperate environment (IHUS and PRUS) did not yield any dictyostelids at all.

For the second experiment, the characterization of parameters for each sampling locations is shown in Table 1. In general, results showed that the location with the highest content of sand was TECR, whereas the lowest content was found in IHUS. Tropical soils (Costa Rica and Mexico) had a higher content of sand $(73.81 \pm 3.35)$ than temperate soils $(68.24 \pm 2.90)$, but no significant differences were found between the two sets. A similar absence of differences was observed for water retention and all three landscape-level parameters when the temperate-tropical comparison was conducted. At the disturbance level, silt values were higher in low disturbance areas than in high or intermediate disturbance areas $(F(2,13)=6.07, P=0.02)$ but no other parameter showed a pattern at this level. Interestingly, water retention showed significant differences $(F(4,13)=4.27$, $P=0.03$ ) between the highest values recorded in the southernmost locations in Costa Rica $(1.32 \pm 0.11 \mathrm{ml} / \mathrm{g}$ soil) and the lowest ones recorded in the northernmost locations in Michigan $(0.70 \pm 0.12 \mathrm{ml} / \mathrm{g}$ soil $)$.

No dictyostelids were observed in the second experiment. The incidence of myxomycetes was found to be greater in temperate samples compared to the tropical samples $(t=-2.45, d . f .=13$, $P=0.01)$ when records from both bacterium types were pooled. When only results obtained with $E$. coli were analyzed, this pattern was maintained, but they were not statistically significant. No differences in the incidence of myxomycetes among disturbance types and across the locations forming the latitudinal gradient were found.

Regarding the enrichment treatments, myxomycetes showed higher incidence values in cornmeal agar $\left(\chi^{2}=8.81\right.$, d.f. $\left.=1, P=0.002\right)$ than in water agar. No myxomycetes were recorded from cultures using the nutrient-rich potato-dextrose agar. In this part of the study, B. subtilis seemed to favor the growth of myxomycetes in comparison with $E$. coli $\left(\chi^{2}=9.43, d . f .=1, P=0.002\right)$.

Table 1 Recorded values for the characterization of soil and landscape parameters based on the sample location used in the second experiment.

\begin{tabular}{lllllllll}
\hline \multicolumn{9}{c}{ Parameters } \\
Location & Origin & \% Sand & \% Silt & \% Clay & WR (ml/g soil) & \% FC & \% HC & Average NDVI \\
\hline IHUS & Michigan & 51.5 & 16.7 & 31.8 & 0.5 & 14.1 & 5.2 & 0.2 \\
PRUS & Michigan & 65.8 & 26.7 & 7.5 & 0.7 & 14.4 & 0.2 & 0.2 \\
IRUS & Michigan & 62.8 & 23.7 & 13.5 & 0.9 & 7.4 & 0.9 & 0.2 \\
BUS & Kentucky & 76.8 & 18.6 & 4.6 & 0.6 & 66.5 & 6.3 & 0.1 \\
EUS & Kentucky & 69.0 & 26.2 & 4.7 & 0.8 & 64.9 & 0.2 & 0.1 \\
PMUS & Kentucky & 66.0 & 33.4 & 0.6 & 1.1 & 91.1 & 0.4 & 0.2 \\
HPUS & Louisiana & 82.5 & 13.0 & 4.5 & 1.1 & 53.3 & 6.5 & 0.3 \\
GUS & Louisiana & 71.5 & 16.8 & 11.7 & 1.0 & 24.1 & 12.2 & 0.2 \\
MMX & Yucatan & 73.9 & 23.6 & 2.5 & 1.1 & 10.6 & 68.8 & 0.1 \\
PMX & Yucatan & 72.4 & 27.4 & 0.2 & 0.7 & 10.1 & 15.6 & 0.2 \\
DMX & Yucatan & 70.3 & 28.7 & 1.0 & 1.0 & 92.5 & 0.4 & 0.3 \\
TACR & Costa Rica & 72.3 & 26.9 & 0.9 & 1.4 & 87.4 & 0.1 & 0.5 \\
OCR & Costa Rica & 67.9 & 27.5 & 4.6 & 1.1 & 27.1 & 0.8 & 0.2 \\
TECR & Costa Rica & 86.1 & 8.2 & 5.7 & 1.5 & 13.1 & 32 & 0.2 \\
\hline
\end{tabular}




\section{Discussion}

The study presented herein was designed to generate baseline data on the potential use of mycetozoans for environmental assessment purposes. In that context, the development and testing of laboratory isolation protocols is relevant given the inequalities on the capacity of protocol execution (by means of the lack of use of a standard protocol in similar studies as well as infrastructural capacity) across different regions in the world.

Results showed that $B$. subtilis was associated with similar incidence values to those yielded using E. coli, particularly for tropical soils and myxomycetes. This finding is interesting for the utilization of alternative protocols because it provides information showing that with the standard "Cavender Method" other bacteria can be used as well. Kenneth Raper (1984) had noted that B. subtilis was suitable for the growth of dictyostelids in laboratory conditions and suggested that the difference with $E$. coli was the generation of more robust reproductive structures with the latter. Similarly, Cohen (1941) found that B. subtilis was a good bacterium for the maintenance of plasmodial cultures of myxomycetes. In this sense, our results support previous reports showing the suitability of $B$ subtilis for mycetozoan isolation. However, such bacterium has also been shown to be highly susceptible to compounds produced by some myxomycetes (see Nakatani et al. 2005) and thus may not be viable for laboratory isolations of all species.

Despite the latter, it is very interesting to note that tropical soils were more productive (albeit not significantly in this study) than temperate ones, for the generation of incidence data obtained from soil samples. Our results are inconclusive and do not demonstrate whether this represents a pattern that deserves more study, or simply represents an artefact of the trials conducted in the present investigation. It is possible that samples sent via air mail may impact the viability of the substrate samples, perhaps as a result of freezing. Studies that have used tropical samples that were shipped to the Unites States typically report lower incidence values. However, in the current study the tropical samples were collected and cultured in the tropics. The temperate samples were sent via airmail and cultured in the tropics and the inverse of the aforementioned pattern was detected.

However, given the fact that our results also showed that the largest differences were obtained for dictyostelids and that there were differences in the incidence values of dictyostelids between temperate and tropical areas, they seem to support the idea that this group of microorganisms is highly prevalent in tropical areas, as it has previously been identified by other researchers (Stephenson \& Landolt 1998). It is worth mentioning, that the tropical locations studied in the present study correspond to the Mesoamerican region, which has been said to be "one of the centres of diversity for the dictyostelid cellular slime moulds" (Swanson et al. 1999).

Regarding the different bait types used in the present study, it is interesting to note the lack of statistical differences, but existence of preference patterns in the generation of data. Even though the first result was obtained herein, it seems that herbaceous baits representing intermediate levels of cellulose to lignin ratios such as leaves, straw or bracts yielded more data. The association between myxomycetes and leafy substrates has been largely documented (e.g. Rollins \& Stephenson 2013, Takahashi 2015, Rojas et al. 2017 for recent works) and dictyostelids have also been found to occur in association with such substrates (e.g. Seephueak \& Petcharat 2014). In the present study, Gmelina sp. sawdust and disaggregated cellulose, the two softest baits, were found to be relatively poor baits for both groups of microorganisms. However, rather than being a result driven by the hardness of the bait, this finding may have been related to the relatively poor (in terms of comparative nutrients) microcosms generated when these baits were used, in comparison with other ones.

The high incidence of myxomycetes in temperate soils is another interesting result recorded in both experiments conducted herein. This may be associated with a more complex vertical distribution of available microhabitats for myxomycetes in tropical areas (see Stephenson et al. 2009) or simply with a more rapid and efficient production of plasmodia in laboratory conditions using temperate soils However, given the limited number of studies on soil myxomycetes, this result demonstrates a modern constraint that may only be elucidated with molecular methods (see Novozhilov et al. 2017). Despite the latter, it is notable that EUS, a location within the coal mining 
landscape in southwestern Virginia, yielded poor results. Given the long history of human activity in the general area surrounding the collecting location, our results may suggest the negative effect of either the habitat disturbance or the mining activity on the microbiological quality of soils. For comparison, other locations in the same general area but located within less disturbed areas yielded significantly higher results (average of $12 \%$ of all positive records for each location versus only $2 \%$ for EUS, $\chi^{2}=14.19$, d.f. $=5, P=0.001$, not shown before). Further evaluations on this particular observation could be conducted using a multiapproach strategy.

For the second experiment, despite documented differences in some of parameters measured during the present investigation, no associations with the incidence of either dictyostelids or myxomycetes were found. In fact, the first group of organisms could not be recorded. Even though this is a limitation of the present study, at least the information on myxomycetes can show some potential lines for future research. For example, the lack of differences in the results according with the landscape-based disturbance classification, may indicate that the level evaluated herein (e.g. incidence from soil samples) could hide more apparent patterns when finer levels, such as quantifying species and their abundance, are used. Previous studies have shown that analyses of myxomycete information in a context of landscape quantification may be sensitive to the scale of experimental design used at both the myxomycete and the landscape level recording (see Rojas et al. 2017).

Even though no correlation was observed between the water retention values and the myxomycete incidence results, it is interesting to note that soils with more capacity to retain water (more sandy and porous in this study) were also observed to yield lower incidental values of myxomycetes. Since no remarkable results were observed at the disturbance types or the latitudinal gradient, our results seem to suggest that site-level characteristics, only seen with a fine characterization of sites, may be a more appropriate strategy to follow in future studies. However, this result may also show that some species of myxomycetes, such as the ones favored with the protocol used herein, may be specific enough for a potential application of the group in a context of environmental assessment. Recent investigations have shown that finer protocols of analysis may show patterns of myxomycete distribution that were previously undocumented (see Dagamac et al. 2017). For example, the results observed for the enrichment treatments in the present study support previous findings (see Wrigley de Basanta \& Estrada-Torres 2017) showing the suitability of the media used. However, since no identification of species was performed, it is impossible to document differences in the species favored to grow in each medium. At this level, such finer protocols could be interesting to implement.

\section{Acknowledgements}

This study was carried out in the context of an international research agreement on microbial ecology between University of Costa Rica and Lincoln Memorial University. Funds were obtained through research code 731-B5-058 and research activity 731-A0-826 from the first institution and from the Cumberland Mountain Research Center at the latter. Additional support was obtained by the first author from the Department of Agricultural and Biosystems Engineering at Michigan State University. We appreciate the collaboration provided in the laboratory by Randall Valverde and Reiner Sibaja of ReForesta.

\section{References}

Atlas D. 2004 - Handbook of Microbiological Media. $3^{\text {rd }}$ edition. CRC Press, Florida.

Clissmann F, Fiore-Donno AM, Hoppe B, Krüger D et al. 2015 - First insight into dead wood protistan diversity: a molecular sampling of bright-spored Myxomycetes (Amoebozoa, slimemoulds) in decaying beech logs. FEMS Microbiology Ecology 91, fiv050.

Cohen A. 1941 - Nutrition of the Myxomycetes. II. Relations between plasmodia, bacteria, and substrate in two-membered culture. Botanical Gazette 103, 205-224. 
Daelher CC, Strong DR. 1996 - Can you bottle nature? The roles of microcosms in ecological research. Ecology 77, 663-664.

Dagamac NHA, Rojas C, Novozhilov YK, Moreno GH et al. 2017 - Speciation in progress? A phylogeographic study among populations of Hemitrichia serpula (Myxomycetes). PLoS ONE 12(4), e0174825.

Douglas TE, Brock DE, Adu-Oppong B, Queller DC et al. 2013 - Collection and cultivation of dictyostelids from the wild. In: Eichinger L, Rivero F. (eds.). Distyostelium discoideum protocols. Springer, New York. Pp 113-124.

Gates DM. 1980 - Biophysical Ecology. Springer-Verlag, New York.

Herrera NA, Rojas C, Franco-Molano AE, Stephenson SL et al. 2011 - Physarella oblonga centered bioassays for testing the biological activity of myxomycetes. Mycosphere 2, 637644.

Lado C, Estrada-Torres A, Wrigley de Basanta D, Schnittler M et al. 2017 - A rapid biodiversity assessment of myxomycetes from a primary tropical moist forest of the Amazon basin in Ecuador. Nova Hedwigia 104, 293-321.

Nakatani S, Kamata K, Sato M, Onuki H et al. 2005 - Melleumin A, a novel peptide lactone isolated from the cultured myxomycete Physarum melleum. Tetrahedron Letters 46, 267-71.

Novozhilov Y, Rollins AW, Schnittler M. 2017 - Ecology and distribution of myxomycetes. In: Stephenson SL, Rojas C (eds.). Myxomycetes: biology, systematics, biogeography and ecology. Academic Press, London. Pp 253-286.

Raper KE. 1984 - The Dictyostelids. Princeton University Press, New Jersey.

Rollins AW, Stephenson SL. 2013 - Myxomycetes associated with grasslands of the western central United States. Fungal Diversity 59, 147-158.

Rojas C, Valverde R, Rollins AW, Murillo-Roos M. 2017 - What can myxomycetes tell us about floricolous microbial systems? Nova Hedwigia 104, 211-220.

Seephueak P, Petcharat V 2014 - The biodiversity of dictyostelid cellular slime molds in rubber tree leaf litter in Southern Thailand. Mycosphere 5, 805-813.

Spiegel FW, Stephenson SL, Keller HW, Moore DL et al. 2004. Mycetozoans. In: Mueller GM, Bills GF, Foster MS (eds). Biodiversity of Fungi: inventory and monitoring methods. Elsevier Academic Press, Massachusetts: 547-576.

Stephenson SL, Landolt JC. 1998. Dictyostelid cellular slime molds in canopy soils of tropical forests. Biotropica 30, 657-661.

Stephenson SL, Schnittler M, Novozhilov Y. 2009 - Myxomycete diversity and distribution from the fossil record to the present. In: Foissner W, Hawksworth D (eds.). Protist Diversity and Geographical Distribution. Springer, New York. Pp 51-67.

Swanson AR, Vadell EM, Cavender JC. 1999 - Global distribution of forest soil dictyostelids. Journal of Biogeography 26, 133-148.

Takahashi K. 2015 - Distribution of myxomycetes on varied leaf litter types in a mixed forest in warm-temperate western Japan. Open Journal of Forestry 5, 686-696.

Tran HTM, Stephenson SL, Hyde KD, Mongkolporn O. 2008 - Distribution and occurrence of myxomycetes on agricultural ground litter and forest floor litter in Thailand. Mycologia 100, 181-190.

Wrigley de Basanta D, Estrada-Torres A. 2017 - Techniques for recording and isolating myxomycetes. In: Stephenson SL, Rojas C (eds.). Myxomycetes: biology, systematics, biogeography and ecology. Academic Press, London: 333-364. 